\title{
Magnetohydrodynamic Boundary Layer Slip Flow and Heat Transfer over a Flat Plate with Heat Generation/Absorption and Viscous Dissipation
}

\author{
Okey Oseloka Onyejekwe \\ Computational Science Program, Addis Ababa University, Arat Kilo campus, Addis Ababa Ethiopia.
}

\begin{abstract}
A numerical study has been carried out on the momentum and heat transfer characteristics of an incompressible magnetohydrodynamic boundary layer slip flow over a flat plate with both viscous and ohmic dissipations. Momentum boundary layer equation takes care of the magnetic field while the ohmic and viscous dissipations are accounted for by the thermal boundary layer equation. The governing equations constitute highly non-linear momentum and thermal boundary layer equations. Both are converted into similarity equations before being solved by the Runge-Kutta-Fehlberg technique with shooting. The results are analyzed for both isothermal and non-isothermal boundary conditions for various combinations of flow and heat transfer parameters. Some of the important findings show that in the absence of both the magnetic and velocity slip parameters, the flow profiles are identical to those of Bhattacharyya et al. [1]. The combined effect of increasing both the magnetic and the slip velocity parameters significantly affects the velocity and the shear stress profiles. An increase of slip parameter results in a decrease in skin friction, whereas an increase in Eckart number enhances viscous dissipation and a consequential temperature rise especially at the boundary. Sometimes this may escalate to a level where a Dirichlet temperature specification is exceeded. Furthermore, the temperature gradient is highly sensitive to prescribed values of Prandtl number and heat generation parameter.
\end{abstract}

Keywords: Magnetohydrodynamic fluid, boundary layer flow, viscous and ohmic dissipations, nonlinear, slips velocity parameter, magnetic parameter

\section{Introduction}

Magnetohydrodynamic flow is a key aspect of computational fluid dynamics. It influences many industrial and flow processes such as crude oil purification, glass manufacturing, generator pumps, metal bearing in contact with fluids, plasma studies, geothermal energy extractions and MHD power generators. This is mainly because the interaction between an electrically conducting fluid and a magnetic field significantly affects the flow field and impacts on the shape of the boundary layer. Some of the early studies in this field include those of Pavlov [2], Anderson[3], Na[4]. MHD flow over a semi infinite flat plate for an incompressible electrically conducting fluid can be found in Alim et al. [5], El-Amin. [6], Mahapatra and Gupta [7]. Ibrahim and Makinde [8] looked at MHD stagnation point flow and heat transfer of Casson nanofluid past a stretching sheet with slip and convective boundary condition. Their results indicate that the skin friction coefficient increased with an increase in Casson parameter and decreased with an increase in velocity ratio parameter. Hayat et al. [9], Hayat et al. [10] carried out similar studies for flow of an electrically conducting fluid over a vertical plate but did not consider nanofluids. Related studies were also carried out by Ishak et al. [11], Watanabe and Pop [11]. Fang and Zang [12] rigorously derived closed form closed-form exact solutions of MHD viscous flows over a shrinking sheet . This work was further extended to include flows over exponential stretching sheet as well as slip effects. (Khan and Sanjayanand[13], Nadeem et al. [14]).

For most of the investigations mentioned above, the conventional no-slip boundary condition is assumed. However this condition is not always admissible. Anderson[15], Aziz [16] studied the effects of slip boundary condition on Newtonian fluids past a stretching sheet. A similar study involving flow over a permeable wall was carried out by Beavers and Joseph [17]. Abas et al. [18] studied the slip effects and heat transfer characteristics for a viscous fluid over an oscillatory stretching surface. Extensive work involving slip effects and temperature dependent viscosity for the diffusion of chemically reactive species on a vertical permeable stretching sheet is reported in Bhattacharyya [19-21].

Of more relevance to the work presented herein are those of Abel et al.[22] who looked into momentum and heat transfer characteristics of an incompressible electrically conducting viscoelastic boundary layer flow over a linear stretching sheet taking into account the effects of transverse magnetic and electric fields as well as ohmic dissipation. And Bhattacharyya et al. [23] who looked into partial slip effects for boundary layer mixed convective flow adjacent to a vertical permeable stretching sheet in a porous medium. From their work, they came to the conclusion that enhancement of buoyancy or mixed convection parameter, increases the 
velocity and decreases the temperature. On the other hand an increase in the slip parameter resulted in an increase in boundary layer thickness. The present work focuses on a detailed numerical investigation of flow and heat transfer characteristics of an MHD flow in an electrically conducting fluid considering the effects of slip, Prandtl and Eckert numbers, as well as heat generation/absorption and viscous dissipation effects. Highly non linear momentum and heat transfer equations are solved numerically adopting Runge-Kutta-Fehlberg method with shooting. Related issues concerning the effects of ignoring viscous thermal dissipation and ohmic heating were also considered. It was observed that relatively high values of the Eckert number, generates more heat due to viscous thermal dissipation. This creates a noticeable influence on the temperature field. As a result viscous dissipation should be accounted for in realistic heat transfer computations especially those involving convective heat transfer.

\section{Mathematical Formulation}

We consider a steady two-dimensional flow of an incompressible laminar boundary layer flow over a semi-infinite flat plate with magnetohydrodynamic effect. It is assumed that the magnetic field is of uniform strength and applied transversely in the flow direction. With regards to the magnetic Reynold number assumption, the induced magnetic field is neglected as well as the electric field due to the polarization of charges. In addition the fluid properties are constants because of the Boussinesq-boundary layer approximations. The cartesian coordinate system is chosen so that $\mathrm{x}=0$, coincides with the leading edge . The plate is parallel to the $\mathrm{x}$-axis and infinitely long downstream with the $\mathrm{y}$-axis normal to it.

In order to fully address the purposes of the work reported herein, we consider two boundary layer equations namely where the flow and energy equations are influenced by the magnetohydrodynamic (MHD), slip and dissipation effects and where none of these are put into consideration. For the latter, the governing equations are written in the usual notation as:

$$
\begin{gathered}
\frac{\partial u}{\partial x}+\frac{\partial v}{\partial y}=0 \\
u \frac{\partial u}{\partial x}+v \frac{\partial u}{\partial y}=v \frac{\partial^{2} u}{\partial x^{2}}+\frac{\sigma B^{2}}{\rho}\left(U_{\infty}-u\right) \\
\rho c_{p}\left(u \frac{\partial T}{\partial x}+v \frac{\partial T}{\partial x}\right)=k \frac{\partial^{2} T}{\partial y^{2}}+Q\left(T-T_{\infty}\right)+\mu\left(\frac{\partial u}{\partial y}\right)^{2}
\end{gathered}
$$

The boundary conditions with partial slip for the velocity are given as:

$$
u=\Psi_{1}(\partial u / \partial y), v=0 \text { at } y=0, u \rightarrow \infty \text { as } y \rightarrow \infty
$$

$T=T_{w} \quad$ at $\quad y=0, \quad T=T_{\infty} \quad$ as $\quad y \rightarrow \infty$

Here $\mathrm{u}$ and $\mathrm{v}$ are the velocity components in the $\mathrm{x}$ and $\mathrm{y}$ directions, $\mathrm{T}$ is the fluid temperature, $\mathrm{k}$ is the thermal conductivity of the fluid, $\mathrm{Q}$ is the heat source or sink, B is the magnetic field in the $\mathrm{y}$ direction and is given by $B(x)=\left(B_{0} /(x)^{1 / 2}\right) U_{\infty}, T_{\infty}, \rho, \sigma$ are the velocity and temperature at infinity, the fluid density and the constant electrical conductivity respectively, $\mu$ is the coefficient of fluid viscosity, $c_{p}$ is the specific capacity at constant temperature of the fluid, $\Psi_{1}=\Psi_{0}\left(\operatorname{Re}_{x}\right)^{1 / 2}$ is the velocity slip factor with $\Psi_{0}$ being the initial velocity slip factor. $\operatorname{Re}_{x}=\left(U_{\infty} x / v\right)$ is the local Reynold's number, $v=(\mu / \rho)$ is the fluid kinematic viscosity, and $T_{w}$ is the wall temperature. We now introduce similarity variables represented by the stream function $\psi(x, y)$ as

$u=\partial \psi / \partial y, v=-\partial \psi / \partial x$. This automatically satisfies the continuity equation and the momentum equation becomes:

$\frac{\partial \psi}{\partial y} \frac{\partial^{2} \psi}{\partial x \partial y}-\frac{\partial \psi}{\partial x} \frac{\partial^{2} \psi}{\partial x \partial y^{2}}=v \frac{\partial^{3} \psi}{\partial y^{3}}+\frac{\sigma B^{2}}{\rho}\left(U_{\infty}-\frac{\partial \psi}{\partial y}\right)$

with boundary conditions represented by 
$\frac{\partial \psi}{\partial y}=\Psi_{1} \frac{\partial^{2} \psi}{\partial y^{2}}, \quad \frac{\partial \psi}{\partial x}=0$ at $y=0, \frac{\partial \psi}{\partial y} \rightarrow U_{\infty}$ as $y \rightarrow \infty$

We introduce the following dimensionless variables for the heat and flow equations

$\theta(\eta)=\frac{T-T_{w}}{T_{w}-T_{\infty}}, \quad \psi=\sqrt{U_{\infty} v x} f(\eta)$

where the similarity variable is defined as $\eta=(y / x)\left(\operatorname{Re}_{x}\right)^{1 / 2}$. The governing differential equations together with their accompanying boundary conditions are then reduced to non-dimensional forms as follows:

$$
\begin{aligned}
& f^{\prime \prime \prime}+0.5 f f^{\prime \prime}+M\left(1-f^{\prime}\right)=0 \\
& \theta^{\prime \prime}+\operatorname{Pr} f \theta+\operatorname{Pr} B \theta+E_{c}\left(f^{\prime \prime 2}\right)=0 \\
& f(\eta)=0, \quad f^{\prime}(\eta)=S f^{\prime \prime}(\eta) \text { at } \eta=0 ; f^{\prime}(\eta) \rightarrow 1 \text { as } \eta \rightarrow \infty \\
& \theta(0)=1, \quad \theta(\infty)=0
\end{aligned}
$$

In the absence of heat dissipation/absorption effect, the heat equation take $s$ the following form:

$\rho c_{p}\left(u \frac{\partial T}{\partial x}+v \frac{\partial T}{\partial y}\right)=k \frac{\partial^{2} T}{\partial y^{2}}+\mu\left(\frac{\partial u}{\partial y}\right)^{2}$

Introducing a dimensionless temperature difference defined as

$$
\theta(\eta)=\frac{T-T_{w}}{U^{2} / 2 c_{p}} \text {, equation (14) becomes }
$$

$\frac{d^{2} \theta}{d \eta^{2}}+0.5 \operatorname{Pr} f \frac{d \theta}{d \eta}=-2 \operatorname{Pr}\left(f^{\prime 2}\right)$

where $\operatorname{Pr}=\mu c_{p} / k$ is the Prandtl number. Other parameters of interest considered in the work reported herein include the heat transfer at the wall computed from Fourier's law; $q_{w}=-k(\partial T / \partial y)_{y=0}$ in transformed variables, this is represented as $q=-k\left(T_{w}-T_{\infty}\right) \sqrt{a / v} \theta^{\prime}(0)$, where $\sqrt{a / v}$ is the characteristic length. Both the constant a and the temperature gradient at the plate $-\theta^{\prime}(0)$ (Nusselt number) are each respectively proportional to the free stream velocity far away from the surface of the flat plate, as well as the rate of heat transfer. Another parameter of interest is the skin-friction coefficient $f^{\prime \prime}(0)$. Overall we have boundaryvalue problems consisting of second-order highly nonlinear simultaneous ordinary differential equations, whose coefficients contain the functions $f, f^{\prime}, \theta$ and $\theta^{\prime}$. In order to solve thee equations numerically we deploy a numerical shooting technique with fifth order Runge-Kutta-Fehlberg (RKF) integration scheme. It is important to choose appropriate representative value for infinity. To implement this, we assume an initial guess value of $\eta_{\infty}$ and solve the problem with particular set of parameters to obtain the dependent variables. The solution process is repeated iteratively for another large value of $\eta_{\infty}$ until two successive values of a given dependent variable differ only by an insignificant value. This guarantees that all numerical solutions approached the asymptotic values correctly. The second and a very important step is to select appropriate initial approximations for the dependent variables. Then the system these initial conditions together with system of first order ordinary differential equations are solved with the RKF integration scheme until the criterion for convergence is achieved. For this work, the step size and the convergence criteria are chosen to be .01 and $10^{-5}$.

\section{Results And Discussion}

A comprehensive numerical calculation is executed for various physical formulation of the flow equation and heat equations and the various impacts of the non-dimensional parameters on the solution profiles In the absence of magnetic field and slip at the boundary $(\mathrm{M}=0, \mathrm{~S}=0)$, the velocity and shear stress profiles of Fig. 1 were found to be identical with those of Bhattacharyya et al. [1]. Fig.2 and Fig. 3 show the contrasting 
influences of the velocity slip parameter on the velocity and shear stress profiles for a fixed value of the magnetic parameter. It is observed that increasing values of the slip velocity parameter causes the velocity profiles to increase monotonically while causing the reverse effect on the shear stress profiles. This is primarily because for the slip condition at the plate, the fluid in contact with the plate, has no longer a zero value. This is reflected in the magnitudes of the velocity profiles as the velocity slip parameter increases. This effect contrasts with the shear stress profiles which decrease with increase in S. In this case, more fluid slide past the plate and the shear stress decreases as the slip parameter increases. This activity is more pronounced in regions very close to the plate, but tends to decrease significantly away from the plate. A close look at regions near the boundary of the Fig. 3 at $\eta=0$ shows that the skin friction coefficient $f^{\prime \prime}(0)$ decreases as the values of the slip velocity increases. Next we study the effect of various values of magnetic parameter on the velocity and shear stress profiles for a fixed value of the slip velocity parameter. Fig. 4 and Fig 5 demonstrate the variations in the velocity and shear stress fields for several values of the magnetic parameter $\mathrm{M}$ for both slip and no slip cases. There is an increase in magnitude for both cases as $\mathrm{M}$ increases. The magnetic force enhances fluid motion in the boundary layer especially for the regions close to the plate. As long as the computed velocity does not exceed that of the free stream, the change in velocity always remains positive $\left(U_{\infty}-u\right) \succ 0$. This enhances the positivity of the momentum equation (equation 2) because the coefficients of this term are also positive and increase with increasing values of the magnetic field. This observation is in agreement with that of Bhattacharyya et al. [1].

Fig. 6 shows the influence of Prandtl number variation for a fixed values of Eckart number $(\mathrm{Ec}=0.5)$, heat generation parameter $(B=0.1)$, magnetic and slip parameters $(M=0.5, S=1.0)$. The thickness of the thermal boundary layer decreases as the Prandtl number increases. This leads to a decrease in the magnitude of temperature profiles. An increase in Prandtl number tesults in a decrease in thermal diffusivity and the fluid energy transfer ability. It also implies an increase in the fluid viscosity which results in a decrease in the flow velocity.

Fig.7 illustrates the temperature flux for the above set of conditions. It confirms the previous observation for the temperature field. In order to dramatize these effects, a flux boundary condition was imposed on the left end of the plate. Fig. 8 shows an easily observable difference in the temperature profiles and further illustrates that a decrease in the thickness of the thermal boundary layer results in an increase in Prandtl number values. For Fig. 9, a high temperature gradient at the wall reflects the effect of the flux boundary condition specified at $\eta=0$. It undergoes a uniform drop for all the profiles irrespective of Prandtl number specification until $\eta \approx 1$. After this, it decouples to display different flux values corresponding to specified values of Prandtl numbers.

The Eckert number or the viscous, thermal dissipation parameter expresses the ratio of the flow's kinetic energy to the boundary layer enthalpy difference and is very useful in heat transfer analysis. Fluid flows with relatively high values of Eckart number $(\mathrm{Ec})$ usually generate a lot of heat. Fig. 10a shows the temperature profiles obtained with variations of Ec for different values of heat and flow parameters ( $\operatorname{Pr}=0.7$, heat generation parameter $\mathrm{B}=0.1$ and $\mathrm{Ec}=0.2,0.5,1.0,1.5$ ). A rise in viscous dissipation results in an increase in energy and a consequential rise in fluid temperature. It can be observed that the highest temperature occurs close to the plate and asymptotically advances to zero towards the free stream area. This is primarily due to the fact that viscous dissipation is felt very close to the wall. Further increase in the values of Eckart number results in high gradients of the temperature field at the boundary. Fig. $10 \mathrm{~b}$ shows that temperatures of this portion of the boundary layer are of such a magnitude that they overshoot the specified boundary temperature due to viscous thermal dissipation. This phenomenon is further enhanced by increasing the Prandtl number.

The effects of various dimensionless parameters on the dimensionless local Nusselt number $-\theta^{\prime}(0)$ are investigated and presented graphically. Fig. 11 is a plot of $-\theta^{\prime}(\eta)$ against the dimensionless space coordinate $\eta$ for different values of Prandtl number ( $\operatorname{Pr}=[10,15,20,30])$ and the following values of dimensionless quantities : $\mathrm{Ec}=[0.5], \mathrm{S}=[0.5]$, heat generaion/absorption $\mathrm{B}=[0.5]$, and magnetic parameter $\mathrm{M}=[0.5]$. It is observed that $-\theta^{\prime}(0)$ increases with a decrease in Prandtl number in order words the rate of heat transfer at the surface increases with decreasing values of Prandtl number. The negative values of the rate of heat transfer indicate that heat is transferred from the plate to the fluid.

Fig. 12 shows the effect of Eckart number Ec on the heat transfer rate. Pr is kept at a value of 0.5 together with all the dimensionless variables mentioned above . Ec is given the values of $[10,15,20,30]$. As mentioned earlier, an increase in Ec results in an increase in temperature distribution. This agrees with the fact that energy is stored in the fluid region as a result of dissipation arising from viscosity and elastic deformation. 
Next, we consider the effects of heat generation and absorption parameter B on the heat transfer rate. In this context, we keep all the other dimensionless quantities constant at 0.5 and assign values of $[-0.5,-1.0,-2,-$ 5.0] to B. Fig. 13 displays the values of the local Nusselt numbers for the different values of Ec numbers. The highest heat transfer rate is recorded for the highest magnitude of the heat generation parameter. We mention in passing that the negative signs in the values of the heat generation parameter indicate the direction of heat flow from the plate to the fluid for the given value of Eckart number Positive values of B are given to reverse the direction of heat generation for the same of values of dimensionless parameters given in the previous example. .Heat absorption takes place in this case and corresponding values of Nusselt numbers are given in Fig. 14. The magnitude and direction of the heat transfer rate agree with the physics.

\section{Conclusions}

Magnetohydrodynamic boundary layer slip flow and heat transfer over a flat plate with heat generation or absorption and viscous dissipation has been studied. The highly nonlinear governing equations were transformed into self-similar form and numerically solved with a shooting technique of a fourth-order RungeKutta-Fehlberg integration scheme. Numerical results obtained herein showed that an increase in the velocity slip parameter results in a decrease in the skin friction and an increase in the fluid velocity. As the velocity slip parameter increases, allowing more fluid to slide through the plate, the magnitude of the velocity profiles close to the plate increases. Various values of the magnetic parameter brought about an increase in the velocity field along the plate and a decrease in the boundary layer thickness. An increase in Prandtl number results in a decrease in both the momentum and thermal boundary layers. On the other hand, an increase in Eckert number enhances the thermal boundary layer. Certain values of Eckart number result in temperatures so high that they overshoot the specified boundary condition as a result of viscous thermal dissipation. Increasing the Newtonian heating parameter facilitates an increase in the rate of heat transfer.

\section{References}

[1] K. BHATTACHARYYA, S. MUKHOPADHYAY, G.C. LAYEK: MHD boundary layer slip flow and heat transfer over a flat plate, Chn. Phs. Lett, 28 (2) (2011), 024701

[2] K.A.PAVLOV: Magnetohydrodynamic flow of an incompressible viscous fluid caused by deformation of a plane surface , Magnitnaya Gidrodinamika 10 (1974), 146-148

[3] H.I. ANDERSON: MHD flow of a viscoelastic fluid past a stretching surface. Acta Mech. 95 (1992), 227-230

[4] T.Y. NA: MHD flow over a moving flat plate. Acta Mech. 116 (1996), 235-238

[5] M.A. ALIM, M.M. ALAM, A.A.MAMUN, M.B. HOSSAIN: Combined effects of viscous dissipation and Joule heating on the coupling of conduction and free convection along a vertical flat plate. Int. Commun. Heat and Mass Transfer 35 (2008), 338-346

[6] M.F. EL-AMIN: Combined effect of viscous dissipation and Joule heating on MHD forced over a non isothermal horizontal cylinder embedded in a saturated porous medium, J. Magn. Magn. Mat. 263 (2003), 337-343

[7] T.R. MAHAPATRA, A.S. GUPTA: Magnetohydrodynamics stagnation point flow towards a stretching sheet, Acta Mech. 152 (2001) , 191-196

[8] W. IBRAHIM, O.D. MAKINDE : Magnetohydrodynamic stagnation point flow and heat transfer of casson nanofluid past a stretching sheet with slip and convective boundary condition, J. Aerosp. Engnr. 29 (2016) , 04015037-1-04015037-9

[9] T. HAYAT, S.A. SHEYZAD, A. ALSAEDI :Soret and Dufour effects on magnetohydrodynamic (MHD) flow of a casson fluid, 33 (2012), 1301-1312

[10] T. HAYAT, M. QASIM, S. MESLOUB : MHD flow and heat transfer over permeable stretching sheet with slip conditions, Int. Jnl. Numer. Fluids, 66 (2011) 963-975

[11] A. ISHAK, K. JAFAR, R. NAZAR, I. POP: MHD stagnation point flow tpwards a stretching sheet, $\quad$ Phys. A. 388 (2009), $3377-$ 3383

[12] T. FANG, J. ZHANG: : Closed form exact solutions of MHD viscous flow over a shrinking sheet, Commun. Nonlinear Sci. Numerical Simulat. , 14 (2009), 2853-2857

[13] S.K. KHAN, E. SANJAYANAND Viscoelastic boundary layer flow and heat transfer over an exponential stretching sheet, Int. Jnl. Heat. Mass Trans., 48 (2005) , 1534-1542

[14] S. NADEEM, R. MEHMOOD, N.S. AKBAR : Combined effects of magnetic field and partial slip on obliquely striking rheological fluid over a stretching surface Jnl. Magn. Magn. Mater. 378 (2015) 457-462

[15] H.I. ANDERSON: Slip flow past a stretching sheet, Acta. Mech., 158 (2002), 121-125

[16] [[16] A. AZIZ: Hydrodynamic and thermal slip flow boundary layer over a flat plate with constant heat flux boundary condition, Commun. Non-Linear Sci. Num. Simul., 15 (2010), 573-580

[17] G.S. BEAVERS, D.D. JOSEPH : Boundary condition at a naturally permeable wall Jnl. Fluid Mech. 30 (1967), 197-207

[18] Z. ABBAS, Y. WANG, T. HAYAT, M. OBERLACK : Slip effects and heat transfer analysis in a viscous fluid over an oscillatory stretching surface, Int. Jnl. Numer. Meth. Fluids, 59 (2009) , 443-458

[19] K. BHATTACHARYYA, G.C. LAYEK: Slip effects on diffusion of chemically reactive species in boundary layer flow due to a vertically stretching sheet with suction or blowing. Chem. Eng. Commun. 198 (2011), 1354-1365

[20] K. BHATTACHARYYA, S. MUKHOPADHYAY, G.C. LAYEK: Effects of partial slip on boundary layer stagnation point flow and heat transfer towards a stretching sheet with temperature dependent fluid viscosity. Acta Techn. 57 (2012), 183-195

[21] K. BHATTACHARYYA, S. MUKHOPADHYAY, G.C. LAYEK: Slip effects on boundary layer stagnation-point flow and heat transfer towards a shrinking sheet, Int. Jnl. Heat and Mass Transfer 54 (2011), 308-313

M. S. ABEL, E. SANJAYANAND, M.M. NANDEPPANAVAR: Viscoelastic MHD flow and heat transfer over a stretching sheet with viscous and ohmic dissipations. Nonlin. Sci. and Num. Simul. 13 (2008) 1808-1821

[23] K. BHATTACHARYYA, M.D. SHARIF, G.C. LAYEK : Effect of partial slip on boundary layer mixed convective flow adjacent to a vertical permeable stretching sheet in porous medium. 58(2013) 27-39 

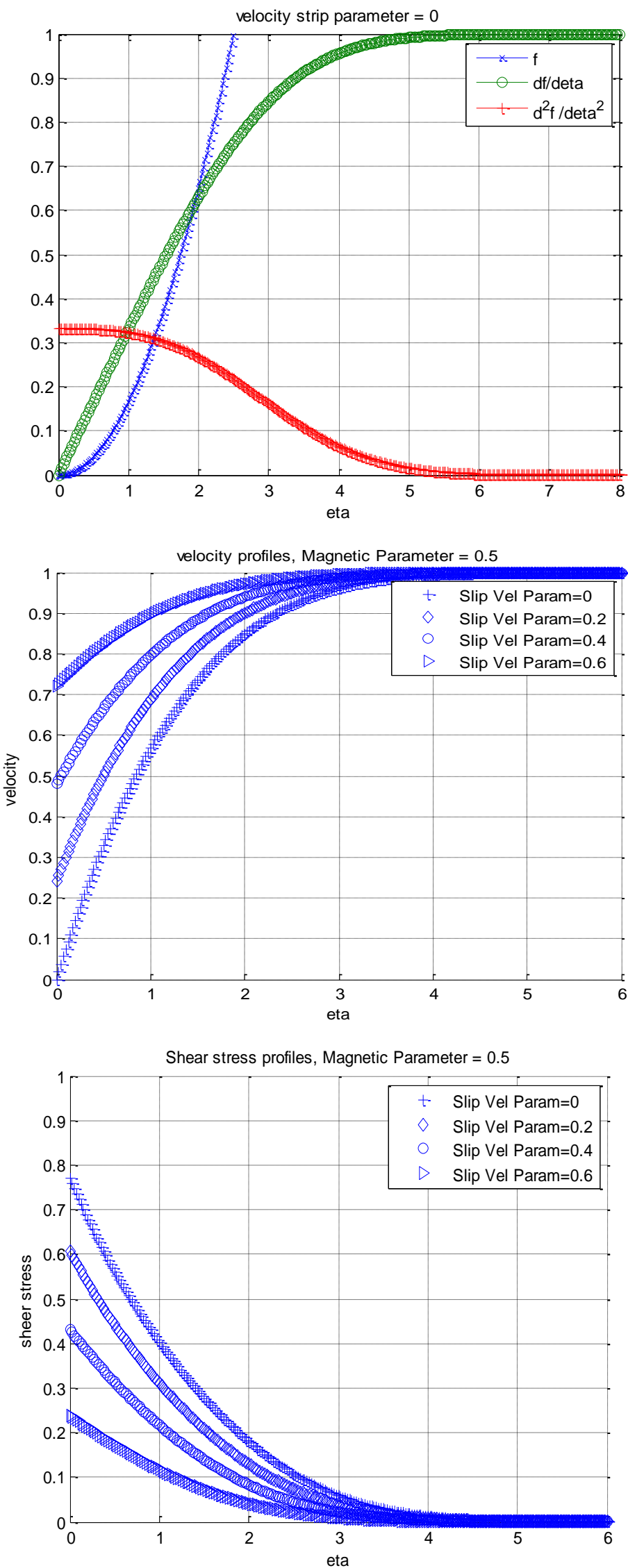

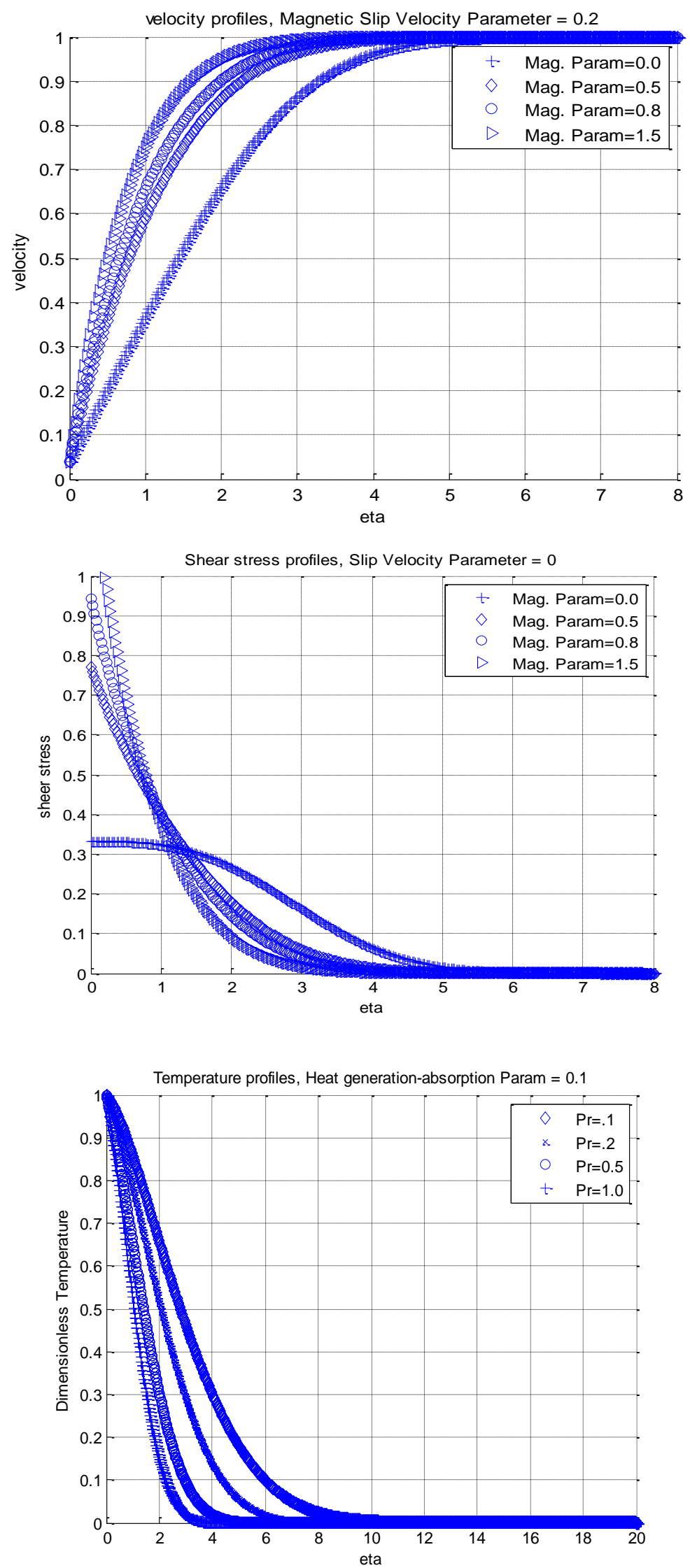

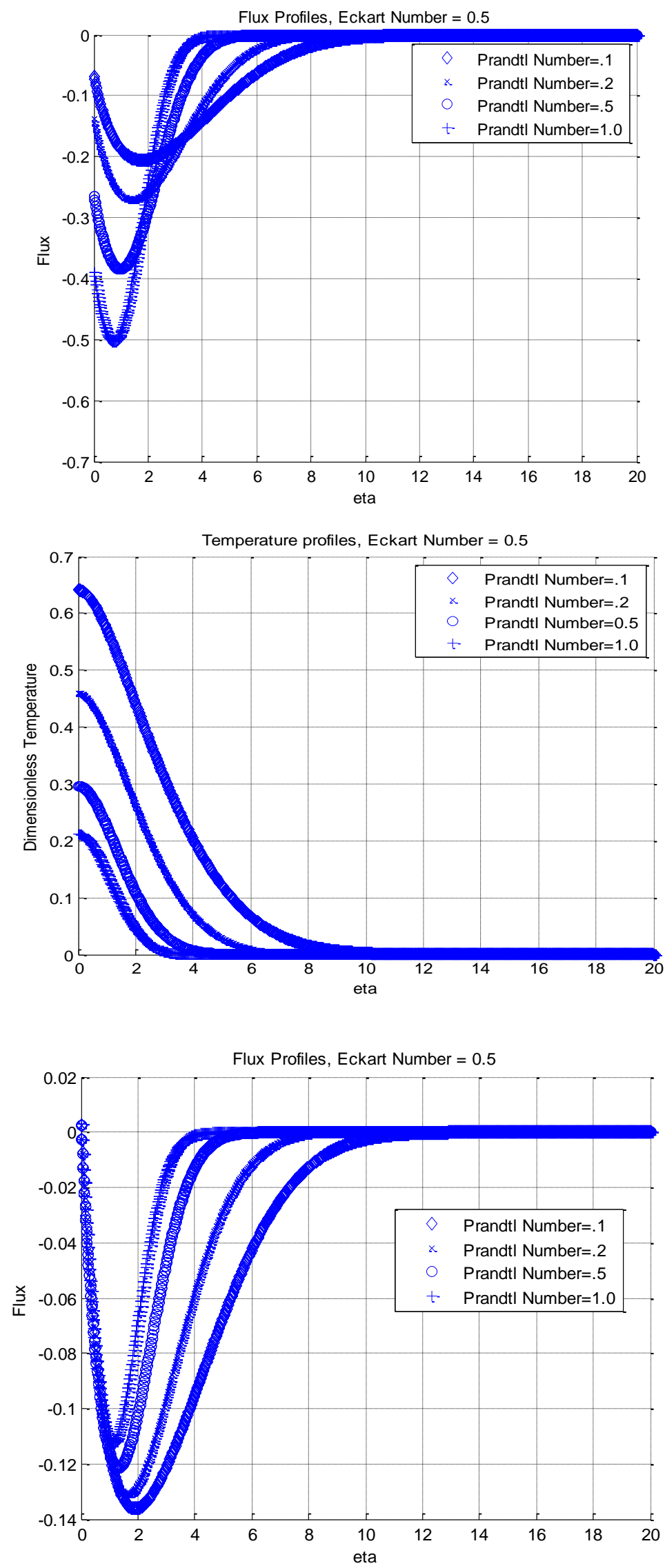

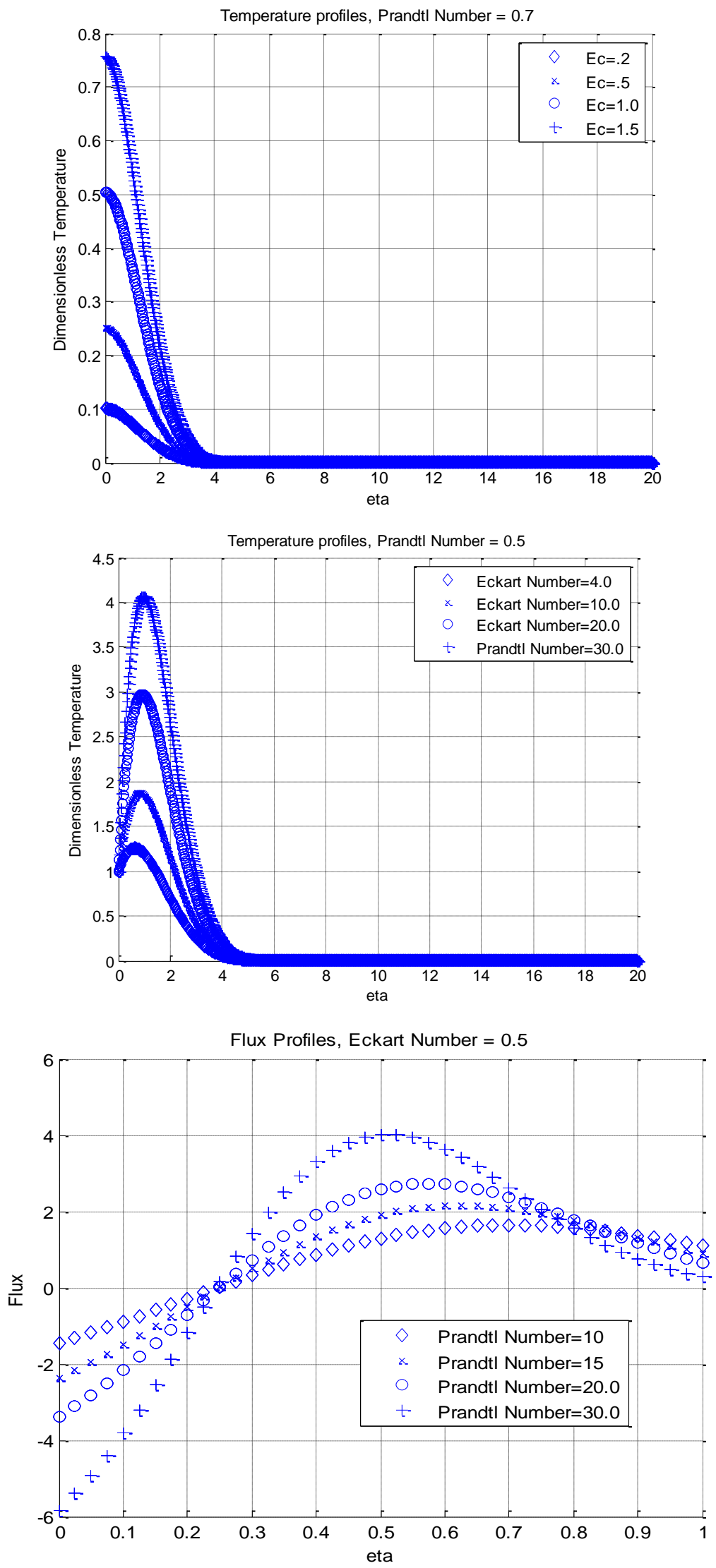

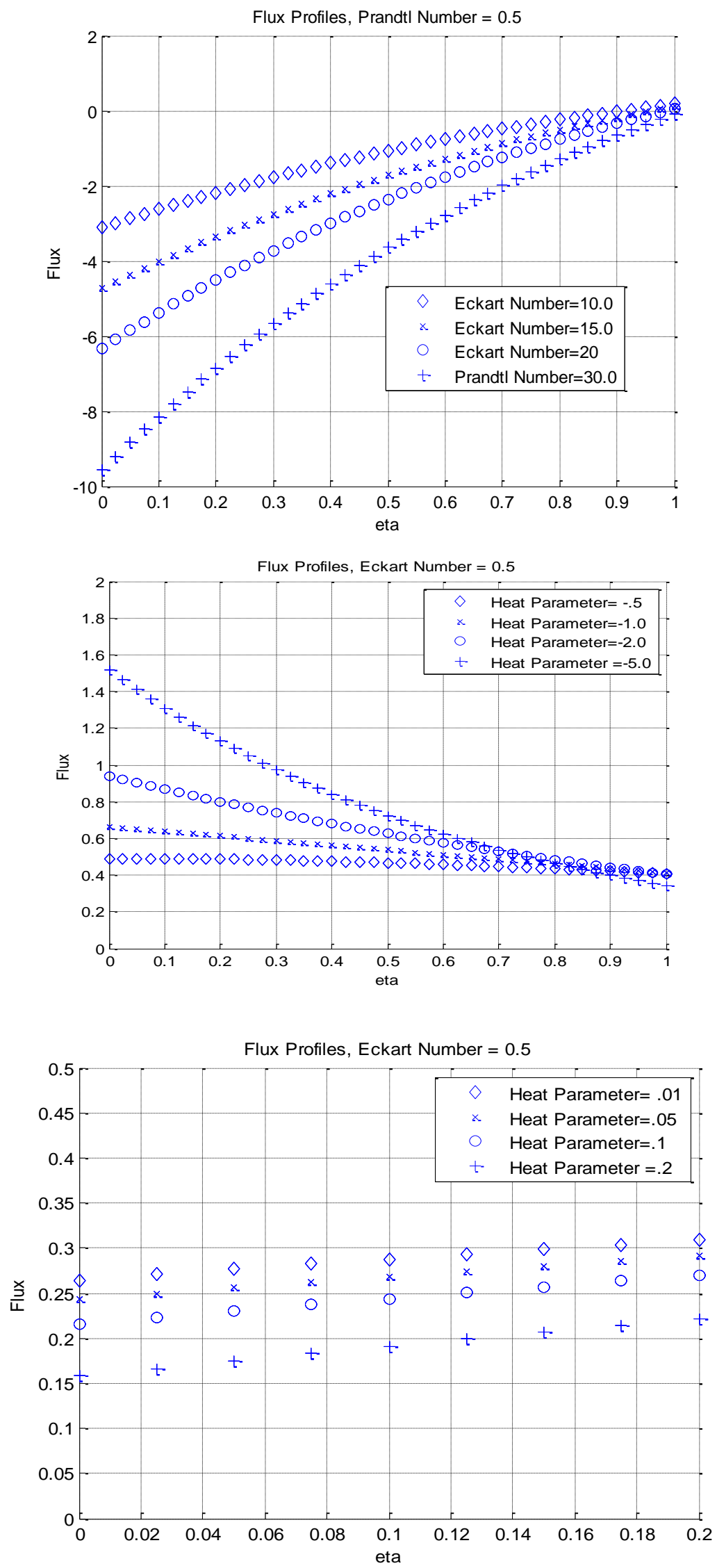


\section{List of Figures}

Fig. 1 Stream function $f$, velocity $f^{\prime}(\eta)$ and shear stress $f^{\prime \prime}(\eta)$ profiles for $\mathrm{M}=0$ and $\mathrm{S}=0$

Fig. 2 Velocity profiles $f^{\prime}(\eta)$ for various values of $\mathrm{S}$

Fig. 3 Shear stress profiles $f^{\prime \prime}(\eta)$ for various values of $\mathrm{S}$

Fig. 4 Velocity profiles $f^{\prime}(\eta)$ for various values of $\mathrm{M}$

Fig. 5 Shear stress profiles $f^{\prime \prime}(\eta)$ for various values of M

Fig. 6 Temperature $\theta(\eta)$ profiles for different values of $\operatorname{Pr}$, and $\mathrm{B}=0.1$

Fig. 7 Temperature gradient $\left(\frac{\partial \theta}{\partial \eta}\right)$ profiles for various values of $\operatorname{Pr}, \mathrm{Ec}=0.5$

Fig. 8 Temperature $\theta(\eta)$ profiles for various values of Pr for a no-flux boundary condition, Ec=0.5

Fig. 9 Temperature gradient $\left(\frac{\partial \theta}{\partial \eta}\right)$ profiles for various values of $\operatorname{Pr}, \mathrm{Ec}=0.5$, for a no flux boundary condition

Fig. 10a Temperature $\theta(\eta)$ profiles for different values of $\mathrm{Ec}, \mathrm{Pr}=0.7$

Fig. 10b Temperature $\theta(\eta)$ profiles overshoot for different values of $\mathrm{Ec}, \operatorname{Pr}=0.7$

Fig. 11 Wall temperature gradient $\left[-\left(\frac{d \theta}{d \eta}\right)_{\eta=0}\right]$ for various values of $\operatorname{Pr}$

Fig. 12 Wall temperature gradient $\left[-\left(\frac{d \theta}{d \eta}\right)_{\eta=0}\right]$ for various values of Ec

Fig. 13 Wall temperature gradient $\left[-\left(\frac{d \theta}{d \eta}\right)_{\eta=0}\right]$ for negative values of $\mathrm{B}$

Fig. 13 Wall temperature gradient $\left[-\left(\frac{d \theta}{d \eta}\right)_{\eta=0}\right]$ for positive values of B 